\title{
BRST symmetry and Darboux transformations in Abelian 2-form gauge theory
}

\author{
Sudhaker Upadhyay* \\ S. N. Bose National Centre for Basic Sciences, \\ Block JD, Sector III, Salt Lake, Kolkata -700098, India.
}

\begin{abstract}
We analyse the constraints of an Abelian 2-form gauge theory using Faddeev-Jackiw symplectic formalism. Further, this theory is treated as a constrained system in the context of Batalin-FradkinVilkovisky formalism to retrieve the BRST symmetry. Using the fields decompositions the effective action for Abelian 2-form gauge theory is written in terms of diagonalized uncanonical part and BRST exact one. The nilpotent BRST and contracting homotopy $\sigma$ closed transformations with field redefinitions are shown as the Darboux transformations used in the Faddeev-Jackiw formalism.
\end{abstract}

\section{INTRODUCTION}

The quantization of non-singular systems is in principle straightforward. On the other hand, the quantization of singular systems (i.e. systems with constraints) is non-trivial. The generalized Hamiltonian dynamics of singular systems was initiated by Dirac [1, 2]. The dynamics of such systems are widely used in investigating theoretical models in contemporary elementary particle physics [3]. Dirac proposed a kind of bracket to quantize these (singular) systems. In the Dirac approach of dealing with singular systems, the dynamical equations involve the variables of the entire phase space, including also unphysical gauge degrees of freedom. However, a symplectic approach to quantize the singular systems has been used by Faddeev and Jackiw (FJ) [4], so-called FJ approach, in which the systematic algorithm involves only the physical (unconstrained) degrees of freedom for arriving at a set of Hamilton equations of motion [5]. In Ref. [6], this algorithm has been shown equivalent to Dirac approach. In FJ approach, the Lagrangian is treated in (symplectic) first order.

Abelian antisymmetric rank-2 tensor field theory is an example of singular system where some of the constraints are not independent and said to be reducible. This theory is the subject of interests in various aspects 7 -14]. For example, Kalb and Ramond has shown that Abelian rank-2 antisymmetric fields interact with classical strings [7], which was further applied to the dual description of Abelian Higgs model [11, 12]. The antisymmetric tensor field appears to couple the gravity or supergravity fields with higher curvature term in four and ten dimensions [13] and complete understanding of these couplings in superstring theories are crucial in order to have anomalies cancellation 14. Abelian 2-form gauge fields have their relevance in M-theory also. In particular, the action for multiple M2-branes was studied via BLG theory [15 19]. The gauge symmetry of this theory was generated by a Lie 3-algebra rather than a Lie algebra. However, this limited the scope of this thoery to two M2-branes. So, this theory was generalized to the ABJM theory 20 24]. The gauge symmetry of this theory is generated by the gauge group $U(N) \times U(N)$. The BRST symmetry of the ABJM theory has also been studied 25 [27]. The ABJM theory has been generalized to the theory of fractional M2-branes [28, 29]. The gauge group of ABJ theory is $U(N) \times U(M)$. Recently, the BRST symmetry of the ABJ theory has also been studied [30 32]. It is shown in [33] at quadratic order of the Lagrangian that the M5-brane theory contains a self-dual two-form gauge field, in addition to the scalars corresponding to fluctuations of the M5-brane in the transverse directions, as well as their fermionic super-partners. The symplectic quantization for Abelian rank-2 antisymmetric tensor field theory has been done in Ref. 34, 35]. However, the Darboux transformations is not studied for the Abelian rank-2 antisymmetric field in the FJ context. This provides a motivation for this present work.

*Electronic address: sudhakerupadhyay@gmail.com; sudhaker@boson.bose.res.in 
Batalin-Fradkin-Vilkovisky (BFV) formulation is a Hamiltonian path integral approach to quantize the constrained systems [36, 37]. In this approach one extends the phase space of the theory by introducing a conjugate momentum for every Lagrange multiplier and a ghost field for every constraint. The induced effective action in extended phase space exhibits a so-called BRST symmetry [38]. However, in FJ approach the phase space is reduced by iteratively solving the constraints and performing the Darboux transformations, until we end up with an unconstrained and canonical Lagrangian. The relation between BFV quantization scheme and the FJ approach for gauged SU(2) WZW model has been established in [39]. We explore it for the reducible gauge theory of Abelian rank-2 tensor field.

In this work we start with the FJ constraint analysis for 2-form gauge theory. The constraints which we found (primary and zero iterated) are exactly same as obtained from Dirac analysis but in more elegant manner. Then, we use BFV approach by extending the phase space to analyse the BRST symmetry of the effective action. Further, the BFV action is written in two terms, the first one is uncanonical term that we would obtain with the FJ method after solving the constraints and the second one is BRST exact term. The BRST transformation and contracting homotopy $\sigma$ closed transformations are calculated for the reducible 2-form gauge theory. Under the fields decompositions these transformations are shown as the Darboux transformation used in FJ formalism.

The paper is organized as follows. In Sec II, we discuss the preliminaries of the FJ symplectic approach of singular system. In Sec. III, we make an analysis to investigate the constraints structure of Abelian rank-2 tensor field theory using symplectic matrix. Then, we stress the BRST-BFV formulation to quantize such reducible gauge theory in sec. IV. Further, in Sec. V, we show that the BRST and contracting homotopy $\sigma$ transformations of 2-form gauge theory are basically Darboux transformations used in FJ symplectic approach. The last section is kept for making concluding remarks.

\section{FADDEEV-JACKIW APPROACH: GENERAL FORMULATION}

In this section we discuss the methodology of FJ approach to quantize the singular systems. In this formalism, we first write the Lagrangian of a singular system into the first-order form as follows:

$$
L(\xi)=a_{i}(\xi) \dot{\xi}^{i}-V(\xi), \quad(i=1,2,3, \ldots . ., n),
$$

where $\xi^{i}$ is called the symplectic variable, $V(\xi)$ is called the symplectic potential. The first-order form can be implemented by introducing some auxiliary variables $\left(a_{i}\right)$ such as the canonical momentum [40]. The Euler-Lagrange equations of motion for Lagrangian (11) can be written as

$$
f_{i j}(\xi) \dot{\xi}^{j}=\frac{\partial V(\xi)}{\partial \xi^{i}} \quad(i=1,2,3, \ldots . ., n),
$$

where $f_{i j}$ is so-called symplectic matrix with following explicit form:

$$
f_{i j}(\xi)=\frac{\partial a_{j}}{\partial \xi^{i}}-\frac{\partial a_{i}}{\partial \xi^{j}}
$$

If matrix $f_{i j}$ is regular (invertible), all symplectic variables can be solved from (2)

$$
\dot{\xi}^{j}=f_{i j}^{-1} \frac{\partial V(\xi)}{\partial \xi^{i}} \quad(i=1,2,3, \ldots . ., n) .
$$

If matrix $f_{i j}$ is singular, there are some constraints in this system. In order to quantize the system with constraints in the FJ method, Barcelos-Neto and Wotzasek [41, 42] proposed the symplectic algorithm to extend the original FJ method [4]. We give a brief description of the symplectic algorithm here. The constraints arising from Eq. (2) are

$$
\Omega_{\alpha}^{(0)}=\left(U_{\alpha}\right)_{i} \frac{\partial V}{\partial \xi^{i}}=0 \quad(\alpha=1,2,3, \ldots, m),
$$


where $U_{\alpha}$ is the zero mode of the symplectic matrix $f, m=n-r(r$ is the rank of $f)$

$$
\left(U_{\alpha}\right)^{T} f=0, \quad(\alpha=1,2,3, \ldots, m) .
$$

Now, we modify our original Lagrangian by introducing the constraint term multiplied with some Lagrange multipliers $\left(v^{\alpha}\right)$ as

$$
L_{\text {mod }}=a_{i}(\xi) \dot{\xi}^{i}-V(\xi)-v^{\alpha} \Omega_{\alpha}^{(0)}, \quad(\alpha=1,2,3, \ldots, m)
$$

and calculate the symplectic matrix with modified Lagrangian density; if there is further constraint in the system then the matrix be- comes singular otherwise it is nonsingular. But, doing iteratively, in the last we get a nonsingular matrix. This means there is no further constraint in the system. So according to the Darboux theorem [43] there exists a coordinate transformation

$$
\begin{gathered}
Q_{1}\left(\xi^{(0)}\right), \ldots \ldots, Q_{m / 2}\left(\xi^{(0)}\right) ; \\
P_{1}\left(\xi^{(0)}\right), \ldots \ldots, P_{m / 2}\left(\xi^{(0)}\right),
\end{gathered}
$$

which transform the first-order Lagrangian given in Eq. (11) into

$$
L^{(0)}=P_{k} \dot{Q}_{k}-V^{(0)}(P, Q), \quad(k=1,2, \ldots \ldots m / 2) .
$$

From the mathematical view, the key of the FJ method is just to construct such a Lagrangian that satisfies the Darboux theorem, and the FJ canonical quantization is established on such a form of the Lagrangian.

In the next section we will treat the Abelian 2-form gauge theory as a singular system and will analyse the constraints using the FJ symplectic approach.

\section{CONSTRAINTS ANALYSIS OF ABELIAN 2-FORM GAUGE THEORY: USING FJ APPROACH}

We start with the Lagrangian density for Abelian free Kalb-Ramond theory in (1+3) dimensions (4D) [7] given by

$$
\mathcal{L}=\frac{1}{12} F_{\mu \nu \rho} F^{\mu \nu \rho},
$$

where the antisymmetric field strength tensor in terms of Kalb-Ramond field $\left(B_{\mu \nu}\right)$ is defined as $F_{\mu \nu \lambda}=$ $\partial_{\mu} B_{\nu \lambda}+\partial_{\nu} B_{\lambda \mu}+\partial_{\lambda} B_{\mu \nu}$. This Lagrangian density is invariant under the following gauge transformation

$$
\delta B_{\mu \nu}=\partial_{\mu} \Lambda_{\nu}-\partial_{\nu} \Lambda_{\mu},
$$

where $\Lambda_{\mu}$ is an arbitrary vector parameter.

This gauge transformation is reducible, since particular choice of vector parameter, i.e.

$$
\Lambda_{\mu}=\partial_{\mu} \varepsilon,
$$

leads to $\delta B_{\mu \nu}=0$.

Now, the canonical momenta corresponding to the fields $B_{0 i}$ and $B_{i j}$, respectively, are calculated as

$$
\Pi^{0 i}=\frac{\partial \mathcal{L}}{\partial \dot{B}_{0 i}}=0
$$

and

$$
\Pi^{i j}=\frac{\partial \mathcal{L}}{\partial \dot{B}_{i j}}=\frac{1}{2} F^{0 i j}
$$


The primary constraint of the theory thus obtained is

$$
\Pi^{0 i} \approx 0 \text {. }
$$

Now, in order to make the Lagrangian density for Abelian 2-form gauge theory in the first order symplectic form, we calculate the following

$$
\Pi^{\mu \nu} \dot{B}_{\mu \nu}-\mathcal{L}=\Pi^{i j} \Pi_{i j}+\frac{1}{12} F_{i j k} F^{i j k}+2 \Pi^{i j} \nabla_{i} B_{0 j} .
$$

So, the first order symplectic version of Lagrangian density given in Eq. (11) is given by

$$
\begin{aligned}
\mathcal{L}^{(0)} & =\Pi^{\mu \nu} \dot{B}_{\mu \nu}-\Pi^{i j} \Pi_{i j}-\frac{1}{12} F_{i j k} F^{i j k}-2 \Pi^{i j} \nabla_{i} B_{0 j}, \\
& =\Pi^{i j} \dot{B}_{i j}-V^{(0)}
\end{aligned}
$$

where $V^{(0)}$ is the symplectic potential with following expression:

$$
V^{(0)}=\Pi^{i j} \Pi_{i j}+\frac{1}{12} F_{i j k} F^{i j k}+2 \Pi^{i j} \nabla_{i} B_{0 j} .
$$

The corresponding symplectic equations of motion can be calculated easily with following relations

$$
f_{i j k \lambda}^{(0)} \dot{\xi}^{k \lambda}=\frac{\partial V^{(0)}(\xi)}{\partial \xi^{i j}},
$$

where

$$
f_{i j k \lambda}^{(0)}=\frac{\partial a_{k \lambda}(\mathbf{y})}{\partial \xi^{i j}(\mathbf{x})}-\frac{\partial a_{i j}(\mathbf{x})}{\partial \xi^{k \lambda}(\mathbf{y})}
$$

The set of symplectic variables are

$$
\xi^{(0)}(x)=\left\{B_{i j}, \Pi_{i j}, B_{o i}\right\} .
$$

The components of symplectic 1-form are calculated as follows:

$$
\begin{aligned}
& a_{B_{i j}}^{(0)}=\frac{\partial \mathcal{L}}{\partial \dot{B}_{i j}}=\Pi^{i j}, \\
& a_{\Pi_{i j}}^{(0)}=\frac{\partial \mathcal{L}}{\partial \dot{\Pi}_{i j}}=0, \\
& a_{B_{0 i}}^{(0)}=\frac{\partial \mathcal{L}}{\partial \dot{B}_{0 i}}=0 .
\end{aligned}
$$

Thus the matrix $f^{(0)}$, whose general form reads

$$
f^{(0)}=\left(\begin{array}{lll}
f_{i k}^{(0) B_{0 i} B_{0 k}} & f_{i k l}^{(0) B_{0 i} B_{k l}} & f_{i k l}^{(0) B_{0 i} \Pi_{k l}} \\
f_{i j k}^{(0) B_{i j} B_{0 k}} & f_{i j k l}^{(0) B_{i j} B_{k l}} & f_{i j k l}^{(0) B_{i j} \Pi_{k l}} \\
f_{i j k}^{(0) \Pi_{i j} B_{0 k}} & f_{i j k l}^{(0) \Pi_{i j} B_{k l}} & f_{i j k l}^{(0) \Pi_{i j} \Pi_{k l}}
\end{array}\right)
$$

is calculated in this case as

$$
f^{(0)}=\left(\begin{array}{ccc}
0 & 0 & 0 \\
0 & 0 & \frac{1}{2}\left(-\delta_{i k} \delta_{j l}+\delta_{i l} \delta_{j k}\right) \\
0 & \frac{1}{2}\left(\delta_{i k} \delta_{j l}-\delta_{i l} \delta_{j k}\right) & 0
\end{array}\right) \delta^{3}(\mathbf{x}-\mathbf{y}) .
$$


It is a singular matrix. The zero mode of this matrix is, $\left(0,0, \nu^{B_{0 i}}\right)$ where $\nu^{B_{0 i}}$ is some arbitrary function. In terms of FJ method [44], using zero-mode, we can obtain constraint

$$
\begin{aligned}
\Omega^{i(0)} & =\left(\nu^{0}\right)_{0 i}^{T} \frac{\partial V^{(0)}}{\partial \xi^{0 i}} \approx 0, \\
& =\nu^{B_{0 i}} \frac{\partial V^{(0)}}{\partial B^{0 i}} \approx 0, \\
& =\nabla_{j} \Pi^{i j} \approx 0,
\end{aligned}
$$

which is zero iterated constraint. This is not independent constraint, since it satisfies the reducibility condition $\nabla_{i} \Omega^{i(0)}=0$. However, it is easy to see that even after calculating the symplectic matrix for modified Lagrangian density with above constraint the zero modes do not lead to any new constraint. Hence, there is no further constraints in the theory.

We end this section by concluding that both the constraints primary and zero iterated are exactly same as obtained in Dirac procedure.

\section{THE EXTENDED ACTION AND BRST SYMMETRY}

In the above section, we obtained two constraints (primary and zero iterated) in the 2-form gauge theory, i.e. $\Pi^{0 i}=0$ and $\nabla_{j} \Pi^{i j}=0$. In this section we discuss the nilpotent BRST symmetry for Abelian rank-2 tensor field theory. To do so, we introduce two pairs of canonically conjugate anticommuting ghosts $\left(\mathcal{C}_{i}, \mathcal{P}_{i}\right)$ and $\left(\overline{\mathcal{C}}_{i}, \overline{\mathcal{P}}_{i}\right)$ corresponding to the above constraints. Further, we need the following pairs of canonically conjugate commuting ghosts $\left(\beta, \Pi_{\beta}\right)$ and $\left(\bar{\beta}, \Pi_{\bar{\beta}}\right)$ which are ghosts of ghosts according to the property of reducibility. The ghosts numbers of these ghost fields are as follows:

$$
\begin{aligned}
g h\left(\mathcal{C}_{i}\right) & =-g h\left(\mathcal{P}_{i}\right)=1, \\
g h\left(\overline{\mathcal{C}}_{i}\right) & =-g h\left(\overline{\mathcal{P}}_{i}\right)=-1, \\
g h(\beta) & =-g h\left(\Pi_{\beta}\right)=2, \\
g h(\bar{\beta}) & =-g h\left(\Pi_{\bar{\beta}}\right)=-2,
\end{aligned}
$$

and they satisfy the following (anti-)commutation relations

$$
\begin{aligned}
\left\{\mathcal{C}_{i}(\mathbf{x}), \mathcal{P}_{j}(\mathbf{y})\right\} & =-i \delta_{i j} \delta^{3}(\mathbf{x}-\mathbf{y}) \\
\left\{\overline{\mathcal{C}}_{i}(\mathbf{x}), \overline{\mathcal{P}}_{j}(\mathbf{y})\right\} & =-i \delta_{i j} \delta^{3}(\mathbf{x}-\mathbf{y}) \\
{\left[\beta(\mathbf{x}), \Pi_{\beta}(\mathbf{y})\right] } & =i \delta^{3}(\mathbf{x}-\mathbf{y}) \\
{\left[\bar{\beta}(\mathbf{x}), \Pi_{\bar{\beta}}(\mathbf{y})\right] } & =i \delta^{3}(\mathbf{x}-\mathbf{y})
\end{aligned}
$$

The phase space is further extended by introducing canonical conjugate pairs $\left(\mathcal{C}_{0}, \mathcal{P}_{0}\right)$ and $\left(\overline{\mathcal{C}}_{0}, \overline{\mathcal{P}}_{0}\right)$ as Lagrange multipliers to the pair $\left(\mathcal{C}_{i}, \mathcal{P}_{i}\right),\left(\overline{\mathcal{C}}_{i}, \overline{\mathcal{P}}_{i}\right)$ and a canonical pair $\left(\varphi, \Pi_{\varphi}\right)$ as Lagrange multiplier to the gauge condition. Hence, the extended action is given by,

$$
\begin{aligned}
S_{e f f} & =\int d^{4} x\left[\Pi^{0 i} \dot{B}_{0 i}+\Pi^{i j} \dot{B}_{i j}+\mathcal{P}^{i} \dot{\mathcal{C}}_{i}+\overline{\mathcal{P}}^{i} \dot{\overline{\mathcal{C}}}_{i}\right. \\
& \left.+\mathcal{P}^{0} \dot{\mathcal{C}}_{0}+\overline{\mathcal{P}}^{0} \dot{\overline{\mathcal{C}}}_{0}+\Pi_{\beta} \dot{\beta}+\Pi_{\bar{\beta}} \dot{\bar{\beta}}+\Pi_{\varphi} \dot{\varphi}-\mathcal{H}_{c}-\{Q, \Psi\}\right],
\end{aligned}
$$

where $\Psi$ is the gauge fixed fermion and $Q$ is the generator of the BRST symmetry. The canonical Hamiltonian density, $\mathcal{H}_{c}$, is calculated as

$$
\mathcal{H}_{c}=\Pi_{i j} \Pi^{i j}+\frac{1}{12} F_{i j k} F^{i j k} .
$$


The expression for BRST charge for Abelian 2-form gauge theory is given by

$$
Q=-2 \nabla_{i} \Pi^{i j} \mathcal{C}_{j}+\Pi_{\varphi} \overline{\mathcal{P}}_{0}-\mathcal{P}_{0} \Pi_{\bar{\beta}}-\overline{\mathcal{P}}^{i} \Pi_{0 i}
$$

which satisfies following algebra

$$
\{Q, Q\}=0, \quad\left\{\mathcal{H}_{c}, Q\right\}=0 .
$$

The ghost numbers of $Q$ and $\Psi$ are as follows:

$$
g h(Q)=1, \quad g h(\Psi)=-1 .
$$

The BRST symmetry transformation can be calculated with following relation

$$
s_{b} \phi=-i[\phi, Q]_{ \pm},
$$

where + is used for fermionic and - for the bosonic nature of generic fields $\phi$. Using the above relation and the expression for BRST charge given in Eq. (32), we calculated the BRST symmetry transformations for fields as follows:

$$
\begin{aligned}
& s_{b} B_{i j}=\left(\nabla_{i} \mathcal{C}_{j}-\nabla_{j} \mathcal{C}_{i}\right), \quad s_{b} B_{0 i}=-\overline{\mathcal{P}}_{i}, \\
& s_{b} \Pi_{\varphi}=0, \quad s_{b} \mathcal{C}_{i}=0, \quad s_{b} \overline{\mathcal{C}}_{i}=\Pi_{0 i}, \quad s_{b} \mathcal{C}_{0}=\Pi_{\bar{\beta}}, \\
& s_{b} \overline{\mathcal{C}}_{0}=\Pi_{\varphi}, \quad s_{b} \varphi=-\overline{\mathcal{P}}_{0}, \quad s_{b} \beta=0, \\
& s_{b} \bar{\beta}=-\mathcal{P}_{0}, \quad s_{b} \Pi_{0 i}=0, \quad s_{b} \Pi_{i j}=0, \\
& s_{b} \mathcal{P}^{i}=2 \nabla_{j} \Pi^{j i}, \quad s_{b} \overline{\mathcal{P}}_{i}=0, \quad s_{b} \mathcal{P}_{0}=0, \\
& s_{b} \overline{\mathcal{P}}_{0}=0, \quad s_{b} \Pi_{\beta}=0, \quad s_{b} \Pi_{\bar{\beta}}=0 .
\end{aligned}
$$

These transformations are nilpotent (i.e. $s_{b}^{2}=0$ ) and symmetry of the effective action given in Eq. (30).

\section{BRST SYMMETRY TRANSFORMATION AS A DARBOUX TRANSFORMATION}

In this section, we study the BRST transformation and the contracting homotopy $\sigma$ transformation for Abelian 2-form gauge theory under the Darboux transformation. For this purpose we first decompose the field $B_{i j}$ into transverse and longitudinal parts as follows

$$
\begin{aligned}
B_{i j} & =B_{i j}^{T}+B_{i j}^{L}, \\
& =\epsilon_{i j k} \nabla_{k} B^{T}+\nabla_{i} B_{j}^{L}-\nabla_{j} B_{i}^{L},
\end{aligned}
$$

where $B_{i j}^{T}=\epsilon_{i j k} \nabla_{k} B^{T}$ and $B_{i j}^{L}=\nabla_{i} B_{j}^{L}-\nabla_{j} B_{i}^{L}$. Then we decompose corresponding momenta $\Pi_{i j}$ into transverse and longitudinal parts as follows

$$
\begin{aligned}
\Pi_{i j} & =\Pi_{i j}^{T}+\Pi_{i j}^{L}, \\
& =\epsilon_{i j k} \frac{\nabla_{k}}{\nabla^{2}} \Pi^{T}+\frac{1}{\nabla^{2}}\left[\nabla_{i} \Pi_{j}^{L}-\nabla_{j} \Pi_{i}^{L}\right],
\end{aligned}
$$

where $\Pi_{i j}^{T}=\epsilon_{i j k} \frac{\nabla_{k}}{\nabla^{2}} \Pi^{T}$ and $\Pi_{i j}^{L}=\frac{1}{\nabla^{2}}\left[\nabla_{i} \Pi_{j}^{L}-\nabla_{j} \Pi_{i}^{L}\right]$. Further, we exploit the relations (36) to solve the field variables $\mathcal{C}_{i}, \overline{\mathcal{P}}_{i}, \Pi_{i j}^{L}$ and $\Pi_{0 i}$ in terms of BRST transformation as follows

$$
\begin{aligned}
\mathcal{C}_{i} & =s_{b} B_{i}^{L}, \\
\overline{\mathcal{P}}_{i} & =s_{b} B_{0 i}, \\
\Pi_{i j}^{L} & =\frac{\nabla_{j}}{2 \nabla^{2}} s_{b} \mathcal{P}_{i}, \\
\Pi_{0 i} & =s_{b} \overline{\mathcal{C}}_{i} .
\end{aligned}
$$


Using the fields decompositions the effective action given in Eq. (30) is written as

$$
\begin{aligned}
S_{e f f} & =\int d^{4} x\left[\Pi_{0 i} \dot{B}^{0 i}+\Pi_{i j}^{T} \dot{B}^{i j T}+2 \frac{\nabla_{i}}{\nabla^{2}} \Pi_{j}^{L} \nabla^{i} \dot{B}^{j L}\right. \\
& -2 \frac{\nabla_{i}}{\nabla^{2}} \Pi_{j}^{L} \nabla^{j} \dot{B}^{i L}+\dot{\mathcal{C}}_{i} \mathcal{P}^{i}+\dot{\overline{\mathcal{C}}}_{i} \overline{\mathcal{P}}^{i} \\
& +\dot{\mathcal{C}}_{0} \mathcal{P}^{0}+\dot{\overline{\mathcal{C}}}_{0} \overline{\mathcal{P}}^{0}+\Pi_{\beta} \dot{\beta}+\Pi_{\bar{\beta}} \dot{\bar{\beta}} \\
& \left.+\Pi_{\varphi} \dot{\varphi}-\mathcal{H}_{c}-\{Q, \Psi\}\right],
\end{aligned}
$$

where the decomposed canonical Hamiltonian density is given by

$$
\begin{aligned}
\mathcal{H}_{c} & =\Pi_{i j}^{T} \Pi^{i j T}+2 \frac{\nabla_{i}}{\nabla^{2}} \Pi_{j}^{L} \frac{\nabla^{i}}{\nabla^{2}} \Pi^{j L} \\
& -2 \frac{\nabla_{i}}{\nabla^{2}} \Pi_{j}^{L} \frac{\nabla^{j}}{\nabla^{2}} \Pi^{i L}+\frac{1}{12} F_{i j k} F^{i j k} .
\end{aligned}
$$

We can easily see that using the symmetry transformations the effective action for Abelian 2-form gauge theory can be recast as

$$
\begin{aligned}
S_{\text {eff }} & =\int d^{4} x\left[\Pi_{i j}^{T} \dot{B}^{i j T}+\Pi_{\beta} \dot{\beta}-\mathcal{H}+s_{b}\left(\overline{\mathcal{C}}^{i} \dot{B}_{0 i}-\mathcal{P}^{i} \dot{B}_{i}^{L}\right.\right. \\
& \left.\left.+\mathcal{C}_{0} \dot{\bar{\beta}}+\overline{\mathcal{C}}_{0} \dot{\varphi}+\frac{1}{4} s_{b} \mathcal{P}_{i} \frac{1}{\nabla^{2}} \mathcal{P}^{i}\right)-\{Q, \Psi\}\right]
\end{aligned}
$$

where

$$
\mathcal{H}=\Pi_{i j}^{T} \Pi^{i j T}+\frac{1}{12} F_{i j k} F^{i j k} .
$$

Hence, we can make the following choice for the gauge fermion

$$
\Psi=i\left(\overline{\mathcal{C}}^{i} \dot{B}_{0 i}-\mathcal{P}^{i} \dot{B}_{i}^{L}+\mathcal{C}_{0} \dot{\bar{\beta}}+\overline{\mathcal{C}}_{0} \dot{\varphi}+\frac{1}{4} s_{b} \mathcal{P}_{i} \frac{1}{\nabla^{2}} \mathcal{P}^{i}\right) .
$$

Exploiting the canonical fields decompositions given in Eqs. (37) and (38), the nilpotent BRST symmetry transformations of Eq. (36) have the following form:

$$
\begin{aligned}
& s_{b} B_{i}^{L}=\mathcal{C}_{i}, \quad s_{b} B_{0 i}=\overline{\mathcal{P}}_{i}, \quad s_{b} \Pi_{\varphi}=0, \\
& s_{b} \mathcal{C}_{i}=0, \quad s_{b} \overline{\mathcal{C}}_{i}=\Pi_{0 i}, \quad s_{b} \mathcal{C}_{0}=\Pi_{\bar{\beta}}, \\
& s_{b} \overline{\mathcal{C}}_{0}=\Pi_{\varphi}, \quad s_{b} \varphi=-\overline{\mathcal{P}}_{0}, \quad s_{b} \beta=0, \\
& s_{b} \bar{\beta}=-\mathcal{P}_{0}, \quad s_{b} \Pi_{0 i}=0, \quad s_{b} \Pi_{i}^{L}=0, \\
& s_{b} \mathcal{P}_{0}=0, \quad s_{b} \mathcal{P}_{i}=2 \nabla^{j} \Pi_{j i}^{L}, \quad s_{b} \overline{\mathcal{P}}_{i}=0, \\
& s_{b} \Pi_{\beta}=0, \quad s_{b} \overline{\mathcal{P}}_{0}=0, \quad s_{b} \Pi_{\bar{\beta}}=0, \\
& s_{b} B_{i j}^{T}=0, \quad s_{b} \Pi_{i j}^{T}=0 .
\end{aligned}
$$

Here we notice that only transverse fields are BRST closed without being BRST exact. Therefore one can show that the functionals of these transverse fields are being used only in classical BRST cohomology. The contracting homotopy $\sigma$ with respect to above BRST operator $s_{b}$ is defined as

$$
\begin{aligned}
& \sigma\left(\mathcal{C}_{i}\right)=B_{i}^{L}, \quad \sigma\left(B_{i}^{L}\right)=0, \quad \sigma\left(\overline{\mathcal{P}}_{i}\right)=B_{0 i}, \\
& \sigma\left(B_{0 i}\right)=0, \quad \sigma\left(\Pi_{0 i}\right)=\overline{\mathcal{C}}_{i}, \quad \sigma\left(\overline{\mathcal{C}}_{i}\right)=0, \\
& \sigma\left(\Pi_{\bar{\beta}}\right)=\mathcal{C}_{0}, \quad \sigma\left(\mathcal{C}_{0}\right)=0, \quad \sigma\left(\Pi_{\varphi}\right)=\overline{\mathcal{C}}_{0}, \\
& \sigma\left(\overline{\mathcal{C}}_{0}\right)=0, \quad \sigma\left(-\overline{\mathcal{P}}_{0}\right)=\varphi, \quad \sigma(\varphi)=0 \\
& \sigma\left(-\mathcal{P}_{0}\right)=\bar{\beta}, \quad \sigma(\bar{\beta})=0, \quad \sigma(\beta)=0, \\
& \sigma\left(2 \nabla^{j} \Pi_{j i}^{L}\right)=\mathcal{P}_{i}, \quad \sigma\left(\mathcal{P}_{i}\right)=0, \\
& \sigma\left(\Pi_{\beta}\right)=0, \quad \sigma\left(B_{i j}^{T}\right)=0, \quad \sigma\left(\Pi_{i j}^{T}\right)=0
\end{aligned}
$$


which is also nilpotent in nature. Further, $\sigma$ operator satisfies the following relation: $\sigma s_{b}+s_{b} \sigma=N$, where $N$ counts the degree in unphysical variables $B_{i}^{L}, \mathcal{C}_{i}, \overline{\mathcal{P}}_{i}, B_{0 i}, \Pi_{0 i}, \overline{\mathcal{C}}_{i}, \Pi_{\bar{\beta}}, \mathcal{C}_{0}, \Pi_{\varphi}, \overline{\mathcal{C}}_{0}, \overline{\mathcal{P}}_{0}, \varphi, \mathcal{P}_{0}, \bar{\beta}, \Pi_{j i}^{L}, \mathcal{P}_{i}$, i.e.

$$
\begin{aligned}
N & =B_{i}^{L} \frac{\partial}{\partial B_{i}^{L}}+\overline{\mathcal{P}}_{i} \frac{\partial}{\partial \overline{\mathcal{P}}_{i}}+\mathcal{C}_{i} \frac{\partial}{\partial \mathcal{C}_{i}}+B_{0 i} \frac{\partial}{\partial B_{0 i}}+\Pi_{0 i} \frac{\partial}{\partial \Pi_{0 i}}+\overline{\mathcal{C}}_{i} \frac{\partial}{\partial \overline{\mathcal{C}}_{i}}+\Pi_{\bar{\beta}} \frac{\partial}{\partial \Pi_{\bar{\beta}}}+\mathcal{C}_{0} \frac{\partial}{\partial \mathcal{C}_{0}} \\
& +\Pi_{\varphi} \frac{\partial}{\partial \Pi_{\varphi}}+\overline{\mathcal{C}}_{0} \frac{\partial}{\partial \overline{\mathcal{C}}_{0}}+\varphi \frac{\partial}{\partial \varphi}+\mathcal{P}_{0} \frac{\partial}{\partial \mathcal{P}_{0}}+\bar{\beta} \frac{\partial}{\partial \bar{\beta}}+\Pi_{j i}^{L} \frac{\partial}{\partial \Pi_{j i}^{L}}+\mathcal{P}_{i} \frac{\partial}{\partial \mathcal{P}_{i}} .
\end{aligned}
$$

It follows that if the functional $\mathcal{G}$ of degree $n \neq 0$ is BRST closed in the unphysical variables,

$$
s_{b} \mathcal{G}=0, \quad N \mathcal{G}=n \mathcal{G},
$$

then it is BRST exact also, i.e. $\mathcal{G}=s_{b}[(1 / n) \sigma \mathcal{G}]$. However, only those BRST closed functionals, which are of degree $n=0$ in the unphysical variables, are not BRST exact, i.e. the functionals of $B_{i j}^{T}, \Pi_{i j}^{T}, \beta, \Pi_{\beta}$ fields.

Therefore, the above BRST and $\sigma$ closed transformations under which the fields transform are basically Darboux transformations used in FJ quantization.

\section{CONCLUSION}

We have considered the Abelian rank-2 antisymmetric tensor field theory (which is a reducible gauge theory) as a singular system and have investigated the constraints involved in the theory using the FJ symplectic approach. Further, we have implemented the BFV formalism in which the scalar potential, $B_{0 i}$, is treated as a full dynamical variable with vanishing conjugate momentum, $\Pi_{0 i}$. According to $\mathrm{BFV}$ formulation the phase space has been extended by introducing a canonical pair of ghost fields for each constraint in the theory. The conserved BRST charge as well as BRST symmetry have been constructed for Abelian 2-form gauge theory within Hamiltonian framework. We have shown that using fields decompositions the effective action for Abelian rank-2 tensor field theory can be written as a sum of an uncanonical term and the BRST exact one. Further, it has been shown that the field redefinitions under which the fields transform into nilpotent BRST and $\sigma$ closed transformations are basically Darboux transformations used in FJ approach.

Further use of similar analysis in the quantum theory of gravity [45 50] and in higher derivative field theory [51] will be interesting. It is also important to mention that within the FJ framework the attempts to derive a non-abelian version of this theory [52] will be exotic.

The path integral corresponding to the FJ quantization method has also been extensively studied under various aspects [53]. So far we have studied the Darboux transformations which appears in the FJ quantization as a symmetry of such path integral. However, it will be interesting to explore the Darboux transformations under which the path integral corresponding to the FJ quantization method is not invariant [54].

[1] P. A. M. Dirac, Lectures on Quantum Mechanics, (Yeshiva Univ. Press, New York, 1964).

[2] P. A. M. Dirac, Can. J. Math. 2, 129 (1950).

[3] M. Henneaux and C. Teitelboim, Quantization of gauge systems ( University Press, Princeton) 1992.

[4] L. D. Faddeev and R. Jackiw, Phys. Rev. Lett. 60, 1692 (1988).

[5] H. J. Rothe and K. D. Rothe, Classical and Quantum Dynamics of Constrained Hamiltonian Systems, (World Scientific, v. 81, 2010).

[6] J. A. García and J. M. Pons, Int. J. Mod. Phys. A 12, 451 (1997).

[7] M. Kalb and P. Ramond, Phys. Rev. D 9, 2273 (1974).

[8] R. K. Kaul, Phys. Rev. D 18, 1127 (1978). 
[9] S. Upadhyay and B. P. Mandal, Mod. Phys. Lett. A 40, 3347 (2010).

[10] S. Upadhyay and B. P. Mandal, Eur. Phys. J. C 72, 2059 (2012).

[11] A. Sugamoto, Phys. Rev. D 19, 1820 (1979).

[12] R. L. Davis and E. P. S. Shellard, Phys. Lett. B 214, 219 (1988).

[13] A. Salam and E. Sezgin, Supergravities in diverse Dimensions (North-Holland and World Scientific, 1989).

[14] M. B. Green, J. H. Schwarz and E. Witten, Superstring Theory (Cambridge Univ. Press, 1987).

[15] J. Bagger and N. Lambert, JHEP 0802, 105 (2008).

[16] J. Bagger and N. Lambert, Phys. Rev. D 77, 065008 (2008).

[17] A. Gustavsson, JHEP 0804, 083 (2008).

[18] M. Faizal, JHEP 1204, 017 (2012).

[19] M. Faizal, arXiv:1303.5477

[20] O. Aharony, O. Bergman, D. L. Jafferis and J. Maldacena, JHEP 0810, 091 (2008).

[21] I. L. Buchbinder, E.A. Ivanov, O. Lechtenfeld, N.G. Pletnev, I.B. Samsonov and B. M. Zupnik, JHEP 0903, 096 (2009).

[22] H. Nastase and C. Papageorgakis, JHEP 1103, 094 (2011).

[23] M. Faizal and D. J. Smith, Phys. Rev. D 85, 105007 (2012).

[24] M. Faizal, Europhys. Lett. 98, 31003 (2012).

[25] M. Faizal, Mod. Phys. Lett. A 27, 1250147 (2012).

[26] M. Faizal Comm. Theor. Phys. 57, 637 (2012).

[27] M. Faizal Phys. Rev. D 84, 106011 (2011).

[28] O. Aharony, O. Bergman and D. L. Jafferis, JHEP 0811, 043 (2008).

[29] J. Kluson, JHEP 0904, 112 (2009).

[30] M. Faizal, JHEP 1301, 156 (2013).

[31] M. Faizal, Nucl. Phys. B 869598 (2013).

[32] M. Faizal, Int. J. Mod. Phys. A28 1350012 (2013).

[33] P. M. Ho and Y. Matsuo, JHEP 0806, 105 (2008).

[34] J. B. Neto and M. B. D. Silva Int. J. Mod. Phys. A 10, 3759 (1995).

[35] R. Banerjee and J. Barcelos-Neto, Annals Phys. 265, 134 (1998).

[36] E. S. Fradkin and G. Vilkovisky, Phys. Lett. B 55, 224 (1975).

[37] I. A. Batalin and G. Vilkovisky, Phys. Lett. B 69, 309 (1977).

[38] C. Becchi, A. Rouet and R. Stora, Annals Phys. 98, 287 (1974).

[39] J. E. Paschalis and P.I. Porfyriadis, Z. Phys. C 73, 557 (1997).

[40] H. Montani and C.Wotzasek, Mod. Phys. Lett. A 8, 3387 (1993).

[41] J. Barcelos-Neto and C. Wotzasek, Mod. Phys. Lett. A 7, 1737 (1992).

[42] J. Barcelos-Neto and C. Wotzasek, Int. J. Mod. Phys. A 7, 4981 (1992).

[43] C. Von Westenholz, Differential Forms in Mathematical Physics (North-Holland, Amsterdam, 1981).

[44] C. Wotzasek, Mod. Phys. Lett., A 8, 2509 (1993).

[45] F. D. Jonghe, J. Paris and W. Troost, Nucl.Phys. B 476, 559 (1996).

[46] M. Faizal, Phys. Lett. B 705, 120 (2011).

[47] M. Faizal J. Phys. A 44, 402001 (2011).

[48] M. Faizal, Found. Phys. 41, 270 (2011).

[49] E. Konishi, Prog. Theor. Phys. 121, 1125 (2009).

[50] G. Esposito, A. Yu. Kamenshchik, I. V. Mishakov and G. Pollifrone, Phys. Rev. D 52, 3457 (1995).

[51] M. Faizal and M. Khan, Eur. Phys. J. C 71, 1603 (2011).

[52] C. S. Chu, S. L. Ko, JHEP 05, 028 (2012).

[53] L. Liao and Y. C. Huang, Phys. Rev. D 75, 025025 (2007).

[54] S. Upadhyay, in progress. 